\title{
Effects of ulinastatin combined with mechanical ventilation on oxygen metabolism, inflammation and stress response and antioxidant capacity of ARDS
}

\author{
MINGXIA JI, TIEJIANG CHEN, BAIMING WANG, MENGYAN CHEN, QIANQIAN DING, LINGCHAO CHEN, \\ YUEJUAN FANG, XIAOFANG YU, YANZHEN CHEN, XIAOHUA WANG, YIYUE HE and YONG JIANG
}

Department of Emergency, Yiwu Central Hospital, Yiwu, Zhejiang 322000, P.R. China

Received August 4, 2017; Accepted November 21, 2017

DOI: $10.3892 /$ etm.2018.6012

\begin{abstract}
Acute respiratory distress syndrome (ARDS) is a disease that seriously threatens human life and health. The aim of the study was to investigate the effects of ulinastatin combined with mechanical ventilation on oxygen metabolism, inflammation and stress response, as well as the antioxidant capacity of ARDS. Eighty patients with ARDS treated in Yiwu Central Hospital from January, 2015 to December, 2016 were enrolled in the present study and divided into the observation $(n=40)$ and control $(n=40)$ groups, using a random number table. The control group was treated with mechanical ventilation, while the observation group, based on treatment of the control group, was treated with ulinastatin for 14 consecutive days as one course of treatment. The changes in the relevant indexes of oxygen metabolism, lung function, time of ventilator treatment, total hospital stay, and St. George's Respiratory Questionnaire (SGRQ) score of the two groups after intervention were compared, and the changes in inflammatory cytokine levels, dopamine receptor-related hormone levels, superoxide dismutase (SOD), malondialdehyde (MDA) and total antioxidant capacity of the two groups before intervention and at 1 and 4 weeks after intervention were compared. After intervention, the arterial blood lactate in the observation group was significantly lower than that in the control group $(\mathrm{P}<0.05)$, the oxygen uptake rate was significantly higher than that in the control group $(\mathrm{P}<0.05)$ and the arterial oxygen content was significantly higher than that in the control group $(\mathrm{P}<0.05)$. In the lung function indexes, the $\mathrm{FEV}_{1}$ and $\mathrm{FEV}_{1} / \mathrm{FVC}$ levels in the observation group were smaller than those in the control group $(\mathrm{P}<0.05)$, the duration of ventilator
\end{abstract}

Correspondence to: Dr Mingxia Ji, Department of Emergency, Yiwu Central Hospital, 519 Nanmen Street, Yiwu, Zhejiang 322000, P.R. China

E-mail: ywzxyy2017@163.com

Key words: ulinastatin, mechanical ventilation, acute respiratory distress syndrome, oxygen metabolism, inflammatory response, stress response, antioxidant capacity treatment was significantly shorter than that in the control group $(\mathrm{P}<0.05)$, and the hospital stay was significantly less than that in the control group $(\mathrm{P}<0.05)$. Prior to intervention, SGRQ scores in the two groups were not statistically significant $(\mathrm{P}>0.05)$. At 1 and 4 weeks after intervention, the SGRQ scores of the observation group were significantly increased to those of the control group $(\mathrm{P}<0.05)$. The tumor levels of necrosis factor- $\alpha$ (TNF- $\alpha$ ), interleukin-6 (IL-6) and CRP were significantly lower than those of the control group $(\mathrm{P}<0.05)$. The levels of adrenaline and norepinephrine were significantly lower than those of the control group $(\mathrm{P}<0.05)$. The levels of MDA, SOD and the total antioxidant capacity were significantly increased to those of control group $(\mathrm{P}<0.05)$. The application of ulinastatin combined with mechanical ventilation in ARDS patients is of great significance in improving the oxygen delivery-consumption balance of body, increasing the lung function, reducing the inflammatory and stress response, and improving the antioxidant capacity.

\section{Introduction}

Acute respiratory distress syndrome (ARDS) is a disease that poses a serious threat to human life and health (1). After onset, it may cause oxygen metabolism imbalance of the body and inflammatory cascade reaction, and activate the body stress response system, thus leading to the impairment or dysfunction of immune system, coagulation system, cardiovascular system, liver and kidney function (2), which eventually leads to the occurrence of multiple organ failure (3). The effective improvement of the clinical treatment of these patients, reduction of the body's inflammatory response and antioxidant response, and improvement of the oxygen delivery-consumption balance of the body are key and difficult points in the treatment of ARDS at present (4).

Ulinastatin is mainly isolated and extracted from the fresh urine of healthy adult males, which, as a kind of glycoprotein, can effectively improve the stability of lysosomal membrane and reduce the synthesis and delivery of lysosomal enzymes, thus scavenging oxygen or hydroxyl radicals, thereby inhibiting the body's inflammatory response $(5,6)$ and improving the body's immune capacity (7). Previously, ARDS patients were treated with mechanical ventilation, but it was mainly 
used to maintain the body's respiratory function without significant effects on the inflammatory and stress response in the body (8). Therefore, in the present study, ulinastatin combined with mechanical ventilation was mainly discussed. The results showed that the technique improved oxygen delivery-consumption balance of body, increased lung function, reduced the inflammatory and stress response, and improved the antioxidant capacity.

\section{Materials and methods}

General data. Eighty patients with ARDS treated in Yiwu Central Hospital from January, 2015 to December, 2016 were selected. The patients were diagnosed via clinical manifestation and pulmonary blood gas analysis. Patients or their authorized persons signed the informed consent and the study was approved by the Ethics Committee of the Yiwu City Centre (Yiwu, China). Patients with acute heart failure, accompanied with hypovolemic shock, other organ system failure, systemic immune system diseases or malignant tumor, whose estimated survival time was within $24 \mathrm{~h}$ or who refused to be enrolled in the study, were excluded.

Patients were divided into the observation $(n=40)$ and control $(n=40)$ groups according to a random number table. In the observation group, there were 24 males and 16 females

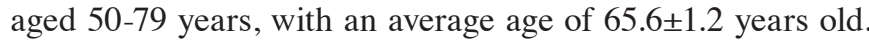
In terms of the causes of disease, there were 17 cases of acute infection, 13 cases of acute trauma, 7 cases of acute poisoning and 3 cases of other causes. The course of disease was 2-48 h with an average of $8.8 \pm 0.3 \mathrm{~h}$. In the control group, there were 25 males and 15 females aged 50-79 years, with an average age of $65.3 \pm 1.3$ years. In terms of the causes of disease, there were 18 cases of acute infection, 12 cases of acute trauma, 8 cases of acute poisoning, and 2 cases of other causes. The course of disease was of $2-48 \mathrm{~h}$ with an average of $8.7 \pm 0.2 \mathrm{~h}$. There were no statistically significant differences in sex, age, causes and course of disease between the two groups $(\mathrm{P}>0.05)$.

Methods. The patients were treated in view of the primary disease, such as improving the body's circulatory function, preventing and treating the disseminated intravascular coagulation (DIC), using effective antimicrobial treatment and infection prevention, strengthening the nutrition intervention, and maintaining water-electrolyte and acid-base balance. The control group was treated with mechanical ventilation. First, the patients were treated with pulmonary re-expansion for 10 consecutive seconds. Then the Respironics 3100B mechanical ventilation instrument (Sensor Medics, Homestead, FL, USA) was connected with the airway pressure of $30 \mathrm{~cm} \mathrm{H}_{2} \mathrm{O}$ under the SIMV mode; the respiratory frequency was set as $5 \mathrm{~Hz}$, the inspiratory/expiratory ratio was set as 1:2 and the fraction of inspired oxygen was set as 80-100\%, and these parameters were decreased step by step. The observation group, based on the treatment of the control group, was treated with the ulinastatin injection (NMPN H20040476; Guangdong Techpool Biochemical Pharmaceutical Co., Ltd., Guangdong, China): 200,000 units were added into $0.9 \%$ $100 \mathrm{ml}$ normal saline for intravenous infusion once every $12 \mathrm{~h}$, and continuous application for 14 days was considered one course of treatment.
Observational indexes. The changes in relevant indexes of oxygen metabolism, lung function, time of ventilator treatment, total hospital stay, and St. George's Respiratory Questionnaire (SGRQ) score of the two groups after intervention were compared, and the changes in inflammatory cytokine levels, dopamine receptor-related hormone levels, superoxide dismutase (SOD), malondialdehyde (MDA) and the total antioxidant capacity of the two groups before intervention and at 1 and 4 weeks after intervention were compared.

Evaluation criteria. Artery blood lactate was detected using whole spectrophotometry (Beckman and Coulter, Brea, CA, USA), and the normal value was $0.5-1.7 \mathrm{mmol} / \mathrm{l}$ (5-15 $\mathrm{mg} /$ day). The oxygen uptake rate mainly reflected the affinity of hemoglobin and oxygen, and the normal value was $25-33 \%$. Arterial oxygen content was detected using enzyme-linked immunosorbent assay (ELISA), and the normal value was 8.55-9.45 $\mathrm{mmol} / \mathrm{l}$. $\mathrm{FEV}_{1}$ was considered the total gas volume within $1 \mathrm{sec}$ of maximal inspiration after maximal expiration. SGRQ score was mainly used for the evaluation of breathing difficulties in the past 4 weeks. There were a total of 76 questions, using the 5-point system and the higher the score, the worse the respiratory function. Inflammatory factors were determined using ELISA, including tumor necrosis factor- $\alpha$ (TNF- $\alpha$ ) (adult's reference value, 1-10 ng/ml), interleukin-1 (IL-1) (adult's reference value, 130-250 ng/ml), IL-6 (adult's reference value, 67.37-150.33 ng/l) and hs-CRP (adult's reference value, $<10 \mathrm{mg} / \mathrm{l})$.The ELISA antibody-sandwich method was used to determine the levels of serum cortisol (adult's reference value, 80-550 nmol/l), epinephrine (adult's reference value, within $480 \mathrm{pmol} / \mathrm{l}$ ) and norepinephrine (adult's reference value, 615-3240 pmol/1). Oxidative stress factors were detected using a 450 full-automatic biochemical detector (Bio-Rad, Hercules, CA, USA), including MDA (normal reference value, 3.52-4.78 $\mathrm{nmol} / \mathrm{ml}$ ), SOD (normal reference value, $0.242-0.620 \mu \mathrm{U} / \mathrm{ml}$ ). The activity of SOD was measured using the Web Standard Tools (WST) method, the level of MDA was measured using the thiobarbituric acid (TBA) method, and the total antioxidant capacity of femoral venous blood was detected using fluorescence recovery after the photobleaching (FRAP) method. The total antioxidant capacity is the sum of large- and small-molecule antioxidases, detected using the FRAP method, and its normal value was $2.34-26.96 \mu \mathrm{U} / \mathrm{ml}$.

Statistical analysis. Statistical Product and Service Solutions (SPSS) \pm 13.0 was used to analyze the data. Measurement data were presented as mean \pm standard deviation (SD). The t-test was used for the comparison of means between the two groups, and the Chi-square test was used for the comparison of rate between the two groups. $\mathrm{P}<0.05$ indicated that the difference was statistically significant.

\section{Results}

Comparisons of oxygen metabolism-related indexes after intervention between the two groups. After intervention, the arterial blood lactate in the observation group was significantly lower than that in the control group $(\mathrm{P}<0.05)$, the oxygen uptake rate was significantly higher than that in the control 
Table I. Comparisons of oxygen metabolism-related indexes after intervention between the two groups (mean \pm SD).

\begin{tabular}{lccc}
\hline Item & Arterial blood lactate $(\mathrm{mmol} / \mathrm{l})$ & Oxygen uptake rate $(\%)$ & Arterial oxygen content (ml/l) \\
\hline Observation group & $2.1 \pm 0.2$ & $33.3 \pm 2.4$ & $156.5 \pm 10.5$ \\
Control group & $3.9 \pm 0.3$ & $25.6 \pm 1.7$ & $89.9 \pm 5.1$ \\
t-value & 31.574 & 16.558 & 36.084 \\
P-value & 0.000 & 0.000 & 0.000 \\
\hline
\end{tabular}

Table II. Comparison of lung function after intervention $($ mean $\pm \mathrm{SD})$.

\begin{tabular}{lcc}
\hline Item & $\mathrm{FEV}_{1}(\%)$ & $\mathrm{FEV}_{1} / \mathrm{FVC}$ \\
\hline Observation group & $58.2 \pm 1.2$ & $41.7 \pm 2.3$ \\
Control group & $42.1 \pm 3.0$ & $61.0 \pm 3.2$ \\
t-value & 31.514 & 30.974 \\
P-value & 0.000 & 0.000 \\
\hline
\end{tabular}

Table III. Comparisons of duration of ventilator treatment and total hospital stay of all the subjects $(d$, mean \pm SD).

\begin{tabular}{lcc}
\hline Item & Ventilator support & Hospital stay \\
\hline Observation group & $3.0 \pm 0.1$ & $31.9 \pm 3.6$ \\
Control group & $4.7 \pm 0.3$ & $42.3 \pm 5.1$ \\
t-value & 34.000 & 10.537 \\
P-value & 0.000 & 0.000 \\
\hline
\end{tabular}

Table IV. Comparison of changes in the SGRQ scores during intervention (point, mean $\pm \mathrm{SD}$ ).

\begin{tabular}{lccc}
\hline Item & $\begin{array}{c}\text { Before } \\
\text { intervention }\end{array}$ & $\begin{array}{c}\text { At 1 week after } \\
\text { intervention }\end{array}$ & $\begin{array}{c}\text { At 4 weeks after } \\
\text { intervention }\end{array}$ \\
\hline $\begin{array}{l}\text { Observation } \\
\text { group }\end{array}$ & $85.9 \pm 2.3$ & $50.3 \pm 1.6$ & $32.7 \pm 1.3$ \\
Control group & $86.0 \pm 2.3$ & $74.6 \pm 2.4$ & $44.3 \pm 2.1$ \\
t-value & 0.194 & 53.281 & 29.705 \\
P-value & 0.846 & 0.000 & 0.000 \\
\hline
\end{tabular}

group $(\mathrm{P}<0.05)$, and the arterial oxygen content was significantly higher than that in the control group $(\mathrm{P}<0.05)$ (Table I).

Comparison of lung function after intervention. In the lung function indexes, the $\mathrm{FEV}_{1}$ and $\mathrm{FEV}_{1} / \mathrm{FVC}$ levels in the observation group were larger than those in the control group $(\mathrm{P}<0.05)$ (Table II).

Comparisons of duration of ventilator treatment and total hospital stay of all the subjects. The duration of ventilator

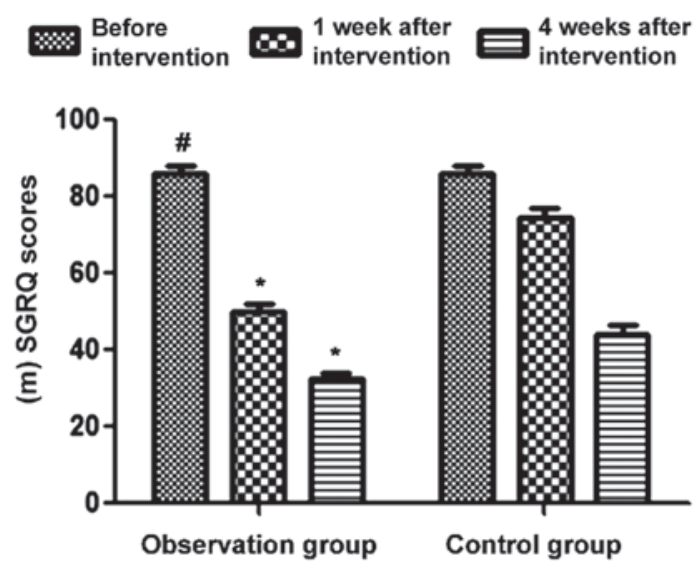

Figure 1. Comparison of changes in St. George's Respiratory Questionnaire (SGRQ) scores during intervention. Prior to intervention, the SGRQ scores in the two groups were not statistically significant $\left({ }^{\#} \mathrm{P}>0.05\right)$. At 1 and 4 weeks after intervention, the SGRQ scores of the observation group were significantly decreased compared to those of the control group $\left({ }^{*} \mathrm{P}<0.05\right)$.

treatment in the observation group was significantly shorter than that of the control group $(\mathrm{P}<0.05)$. In addition, the hospital stay was significantly less in the observation group than that in the control group $(\mathrm{P}<0.05)$ (Table III).

Comparison of changes in SGRQ scores during intervention. Prior to intervention, the SGRQ scores in the two groups were not statistically significant $(\mathrm{P}>0.05)$. At 1 and 4 weeks after intervention, the SGRQ scores of the observation group were significantly decreased compared to those of the control group $(\mathrm{P}<0.05)$ (Table IV and Fig. 1).

Changes in inflammatory cytokine levels during intervention. There were no statistically significant differences in the levels of TNF- $\alpha$, IL- 6 and CRP between the two groups before intervention $(\mathrm{P}>0.05)$. At 1 and 4 weeks after intervention, the levels of TNF- $\alpha$ in the observation group were $113.1 \pm 14.5$ and $105.0 \pm 5.0 \mathrm{ng} / \mathrm{l}$, the levels of IL- 6 were $110.4 \pm 7.4$ and $79.0 \pm 3.1 \mathrm{ng} / \mathrm{l}$, and the levels of CRP were $7.20 \pm 0.3$ and $6.0 \pm 0.1 \mathrm{mg} / 1$, which were significantly lower than those in control group, except CRP levels at 1 week $(\mathrm{P}<0.05)$ (Fig. 2).

Changes in dopamine receptor-related hormone levels of the two groups during intervention. There were no statistically significant differences in adrenaline and norepinephrine levels between the two groups before intervention $(\mathrm{P}>0.05)$. At 1 and 4 weeks after intervention, the levels of adrenaline in the observation group were $103.2 \pm 6.4$ and $58.0 \pm 2.1 \mathrm{pmol} / 1$, respectively, and the levels of norepinephrine were $174.7 \pm 17.9$ 


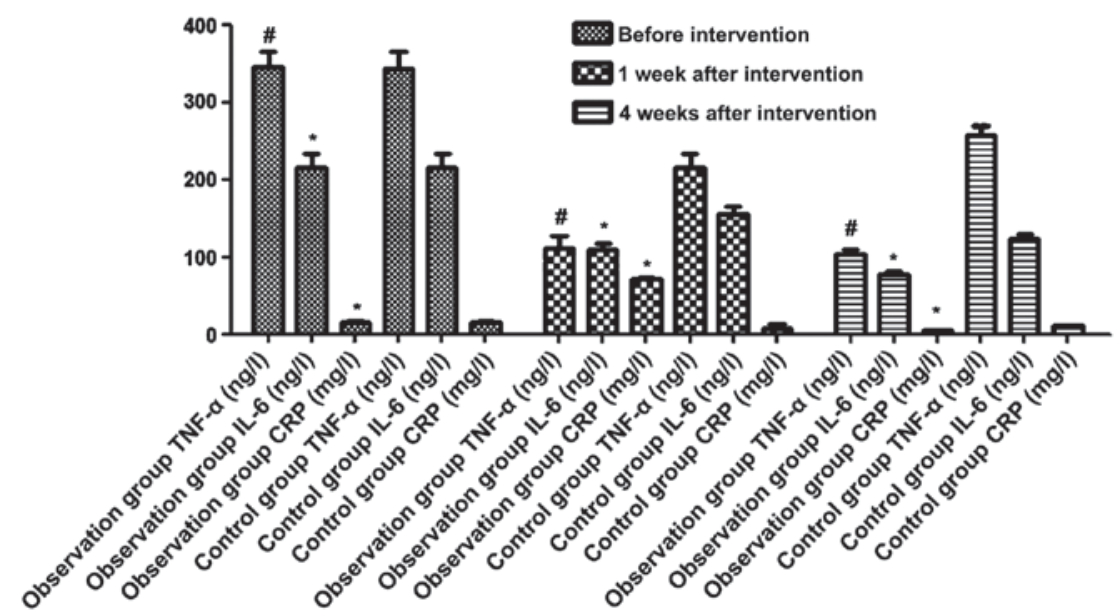

Figure 2. Changes in inflammatory cytokine levels during intervention. There are no statistically significant differences in the levels of tumor necrosis factor- $\alpha$ (TNF- $\alpha$ ), interleukin-6 (IL-6) and CRP between the two groups before intervention $\left({ }^{*} \mathrm{P}>0.05\right)$. At 1 and 4 weeks after intervention, the levels of TNF- $\alpha$, IL- 6 and CRP in observation group are significantly lower than those in control group ("P<0.05).

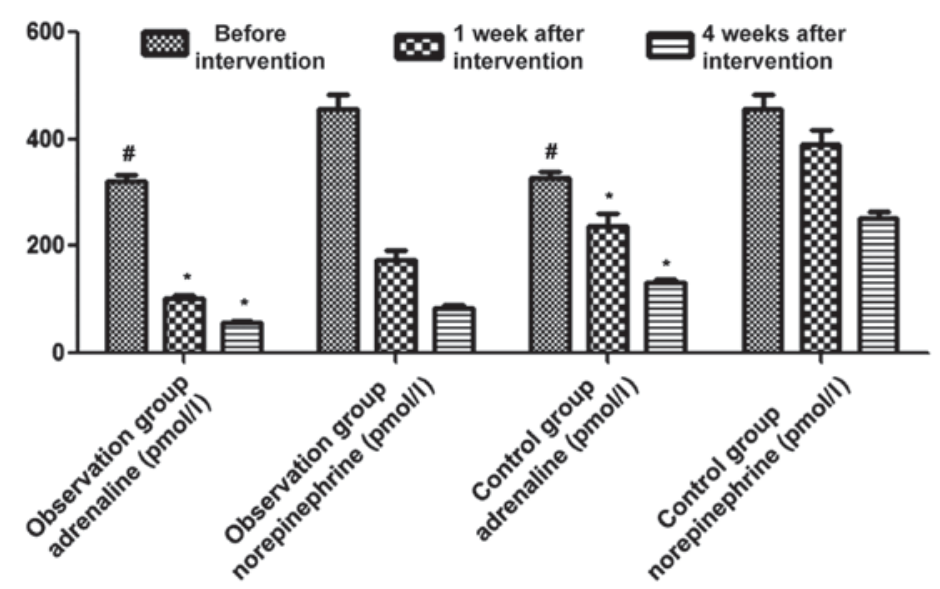

Figure 3. Changes in dopamine receptor-related hormone levels of the two groups during intervention. There are no statistically significant differences in adrenaline and norepinephrine levels between the two groups before intervention $\left({ }^{\#} \mathrm{P}>0.05\right)$. At 1 and 4 weeks after intervention, the levels of adrenaline and norepinephrine in the observation group were significantly lower than those in the control group ( $\mathrm{P}<0.05)$.

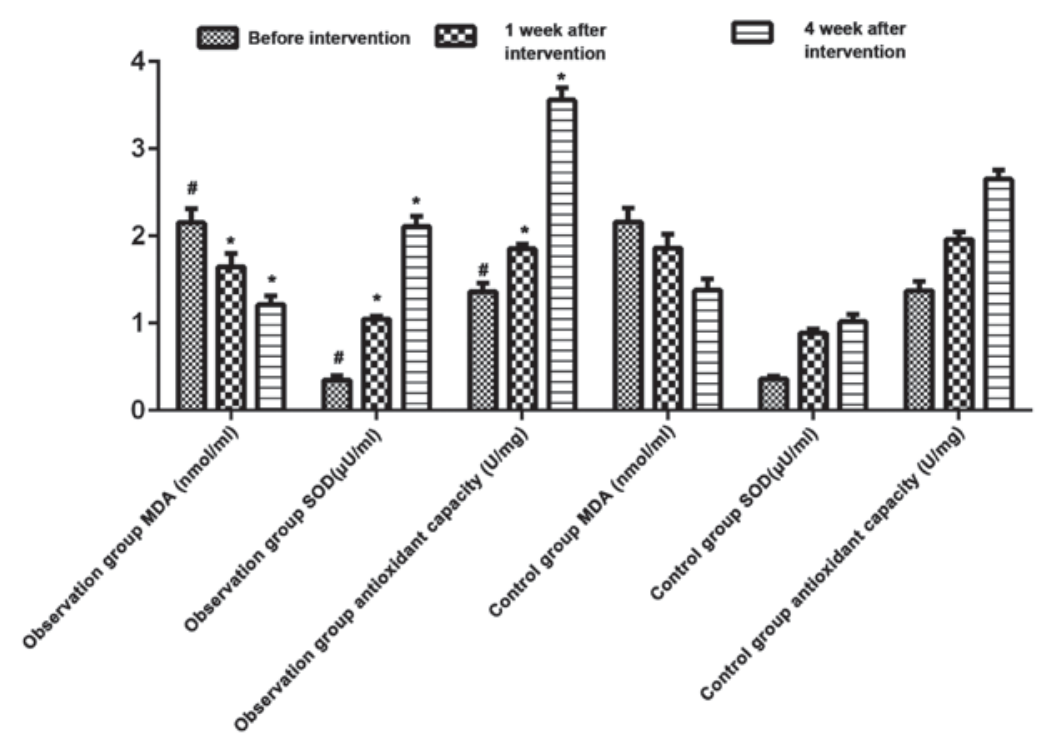

Figure 4. Comparisons of superoxide dismutase (SOD), malondialdehyde (MDA) and total antioxidant capacity between the two groups during intervention. There were no statistically significant differences in MDA, SOD and total antioxidant capacity between the two groups before intervention $\left({ }^{\sharp} \mathrm{P}>0.05\right)$. At 1 and 4 weeks after intervention, the levels of MDA, SOD was lower and total antioxidant capacity in the observation group were better to those in the control group ( $(\mathrm{P}<0.05)$. 
and $84.5 \pm 6.1 \mathrm{pmol} / \mathrm{l}$, respectively, which were significantly lower than those in the control group $(\mathrm{P}<0.05)$ (Fig. 3).

Comparisons of SOD, MDA, and the total antioxidant capacity between the two groups during intervention. There were no statistically significant differences in MDA, SOD and the total antioxidant capacity between the two groups before intervention $(\mathrm{P}>0.05)$. At 1 and 4 weeks after intervention, the levels of MDA in the observation group were $1.65 \pm 0.15$ and $1.21 \pm 0.11 \mathrm{nmol} / \mathrm{ml}$, the levels of SOD were $1.36 \pm 0.11$ and $0.35 \pm 0.05 \mu \mathrm{U} / \mathrm{ml}$, and the total antioxidant capacity was $2.11 \pm 0.12$ and $3.56 \pm 0.14 \mathrm{U} / \mathrm{mg}$, which were significantly increased to those in the control group $(\mathrm{P}<0.05)$ (Fig. 4).

\section{Discussion}

ARDS is caused by a variety of primary diseases, which mainly manifest in progressive hypoxia and respiratory failure (9). Its pathological feature is primarily diffuse alveolar epithelial cell and pulmonary microvascular endothelial cell damage, mostly accompanied with severe pulmonary edema and the formation of pulmonary hyaline membranes. With disease progression, the lung volume is significantly reduced, lung compliance is decreased, and ventilation/blood flow ratio is out of balance (10). The imbalance of oxygen metabolism, inflammatory response and antioxidant capacity disorder are important links in the occurrence and development of ARDS (11). High-frequency oscillatory ventilation ventilates through the high airway positive pressure circuit formed by the high speed continuous air flow, which is repeatedly superimposed by the shock wave formed by the piston device, thus reducing the gas and $\mathrm{CO}_{2}$ retention in the normal breathing support (12), thereby significantly improving the respiratory support effect. However, single application of mechanical ventilation is mainly used to improve the respiratory function of patients with ARDS, but has no significant effect on the body's inflammatory response and stress response (13). Thus, ulinastatin and mechanical ventilation were combined.

Findings of the present study showed that after intervention, the arterial blood lactate in the observation group was significantly lower than that in the control group, the oxygen uptake rate was significantly higher than that in the control group, and the arterial oxygen content was significantly higher than that in the control group. In the lung function indexes, $\mathrm{FEV}_{1}$ and FEV1/FVC level in the observation group were larger than those in the control group, suggesting that the application of ulinastatin in ARDS patients can significantly improve the body's oxygen supply-demand balance, improve the lung function, reduce the lactic acid accumulation and improve the patients' prognosis. In addition, the duration of ventilator treatment was significantly shorter than that in the control group and the hospital stay was significantly less than that in the control group. At 1 and 4 weeks after intervention, the SGRQ scores of the observation group were significantly increased compared to those of the control group, further suggesting that the application of ulinastatin in ARDS patients is important for shortening the duration of ventilator treatment and hospital stay and improving the overall respiratory function of patients. At the same time, the levels of TNF- $\alpha$, IL- 6 and CRP were significantly lower than those of the control group at 1 and 4 weeks after intervention, suggesting that the application of ulinastatin in ARDS patients can decrease the body's inflammatory cytokines. Finally, at 1 and 4 weeks after intervention, the levels of adrenaline and norepinephrine' were significantly lower than those of the control group, while the level of SOD and total antioxidant capacity were significantly increased compared to those of the control group, indicating that the application of ulinastatin in ARDS patients has a positive significance in reducing the oxygen-free radical damage and decreasing the stress response.

Ulinastatin, as a kind of broad-spectrum protease inhibitor, can effectively inhibit the activity of inflammatory factors and reduce their levels, thus playing a role in protecting the body organs and system (14). Its intravenous administration can effectively inhibit a variety of cell proteolytic enzymes in the body (15), reduce the damage of hydrolase to normal tissues, stabilize the intracellular lysosomal membrane, and reduce the body peroxidation reaction by inhibiting the production of peroxide, thus inhibiting the inflammatory response (16). In addition, ulinastatin can inhibit the proteolytic enzyme activity widely and improve body microcirculation and tissue perfusion functions (17). The stability of intracellular lysosomal membrane (18) can help improve the organelle mitochondrial activity, improve cell respiration (19), enhance cell elastinase activity, and remove the body oxygen or hydroxyl-free radicals (20).

In conclusion, the application of ulinastatin combined with mechanical ventilation in ARDS patients is of great significance in improving the oxygen delivery-consumption balance of the body, increasing lung function, reducing the inflammatory and stress response and improving the antioxidant capacity.

\section{Competing interests}

The authors declare that they have no competing interests.

\section{References}

1. Wang L, Huang X, Kong G, Xu H, Li J, Hao D, Wang T, Han S, Han C, Sun Y, et al: Ulinastatin attenuates pulmonary endothelial glycocalyx damage and inhibits endothelial heparanase activity in LPS-induced ARDS. Biochem Biophys Res Commun 478: 669-675, 2016.

2. Qiu Y, Lin J, Yang Y, Zhou J, Gong LN, Qin Z and Du L: Lack of efficacy of ulinastatin therapy during cardiopulmonary Bypass surgery. Chin Med J (Engl) 128: 3138-3142, 2015.

3. Sun R, Li Y, Chen W, Zhang F and Li T: Total ginsenosides synergize with ulinastatin against septic acute lung injury and acute respiratory distress syndrome. Int J Clin Exp Pathol 8: 7385-7390, 2015

4. Leng YX, Yang SG, Song YH, Zhu X and Yao GQ: Ulinastatin for acute lung injury and acute respiratory distress syndrome: A systematic review and meta-analysis. World J Crit Care Med 3: 34-41, 2014.

5. Yuan S: Drugs to cure avian influenza infection--multiple ways to prevent cell death. Cell Death Dis 3: 4835-4839, 2013.

6. Sekine Y and Ko E: The influence of intraoperative oxygen inhalation on patients with idiopathic pulmonary fibrosis. Masui 60: 307-313, 2011 (In Japanese).

7. Zhou LW, Wang YL, Yan XT and He XH: Urinary trypsin inhibitor treatment ameliorates acute lung and liver injury resulting from sepsis in a rat model. Saudi Med J 29: 368-373, 2008.

8. Nakanishi K, Takeda S, Sakamoto A and Kitamura A: Effects of ulinastatin treatment on the cardiopulmonary bypass-induced hemodynamic instability and pulmonary dysfunction. Crit Care Med 34: 1351-1357, 2006 
9. Inoue K, Takano H, Yanagisawa R, Sakurai M, Shimada A, Sato H, Kato Y and Yoshikawa T: Antioxidative role of urinary trypsin inhibitor in acute lung injury induced by lipopolysaccharide. Int J Mol Med 16: 1029-1033, 2005.

10. Inoue K, Takano H, Yanagisawa R, Sakurai M, Shimada A, Yoshino S, Sato H and Yoshikawa T: Protective role of urinary trypsin inhibitor in acute lung injury induced by lipopolysaccharide. Exp Biol Med (Maywood) 230: 281-287, 2005.

11. Ito K, Mizutani A, Kira S, Mori M, Iwasaka H and Noguchi T: Effect of ulinastatin, a human urinary trypsin inhibitor, on the oleic acid-induced acute lung injury in rats via the inhibition of activated leukocytes. Injury 36: 387-394, 2005.

12. Gaudry S, Tuffet S, Lukaszewicz AC, Laplace C, Zucman N, Pocard M, Costaglioli B,Msika S, Duranteau J, Payen D, et al: Prone positioning in acute respiratory distress syndrome after abdominal surgery: a multicenter retrospective study: SAPRONADONF (Study of Ards and PRONe position After abDOmiNal surgery in France). Ann Intensive Care 7: 21, 2017.

13. FowlerIii AA,KimC,Lepler L,MalhotraR,Debesa O,NatarajanR, Fisher BJ, Syed A, DeWilde C, Priday A, et al: Intravenous vitamin $\mathrm{C}$ as adjunctive therapy for enterovirus/rhinovirus induced acute respiratory distress syndrome. World J Crit Care Med 6: 85-90, 2017.

14. Albert M, Corsilli D, Williamson DR, Brosseau M, Bellemare P, Delisle S, Nguyen AQ and Varin F: Comparison of inhaled milrinone, nitric oxide and prostacyclin in acute respiratory distress syndrome. World J Crit Care Med 6: 74-78, 2017.
15. Wang T, Hou W and Fu Z: Preventative effect of OMZ-SPT on lipopolysaccharide-induced acute lung injury and inflammation via nuclear factor-kappa B signaling in mice. Biochem Biophys Res Commun 485: 284-289, 2017.

16. Ni YN, Luo J, Yu H, Wang YW, Hu YH, Liu D, Liang BM and Liang ZA: Can body mass index predict clinical outcomes for patients with acute lung injury/acute respiratory distress syndrome? A meta-analysis. Crit Care 21: 36, 2017.

17. Holopainen S, Hytönen MK, Syrjä P, Arumilli M, Järvinen AK, Rajamäki M and Lohi H: ANLN truncation causes a familial fatal acute respiratory distress syndrome in Dalmatian dogs. PLoS Genet 13: e1006625, 2017.

18. Bolaki M, Amargianitakis V, Georgopoulos D, Guervilly C and Papazian L: Effects of neuromuscular blockers on transpulmonary pressures in moderate to severe acute respiratory distress syndrome. Intensive Care Med 43: 600-601, 2017.

19. Takeshima M, Ishikawa $H$ and Shimizu T: Acute respiratory distress syndrome and lamotrigine: A case report. Psychosomatics 18: 331-382, 2016.

20. Mohamed HS and Meguid MM: Effect of nebulized budesonide on respiratory mechanics and oxygenation in acute lung injury/acute respiratory distress syndrome: Randomized controlled study. Saudi J Anaesth 11: 9-14, 2017.

(i) () $\ominus$ This work is licensed under a Creative Commons EY NG No Attribution-NonCommercial-NoDerivatives 4.0 International (CC BY-NC-ND 4.0) License. 